subjects (neuromotor score, NMS $\geq 2$, or Bayley Scales of Infant Development II or III MDI $<70$ or cognitive score $<85$ ).

Chorioamnionitis (42 subjects) was associated with a lower risk of moderate-severe brain injury (OR 0.3; 95\%CI 0.1-0.7; p=0.003), and trended toward lower risk of adverse neurodevelopment. Infant infection (32 subjects) trended toward association with moderatesevere injury (OR 1.6; 95\%CI 0.8-3.5; $\mathrm{p}=0.2$ ), and was significantly associated with an abnormal NMS (OR 3.4; 95\%CI 1.2-10.2; $\mathrm{p}=0.03$ ) but not cognitive outcome. After adjusting for hypothermia and severity of encephalopathy, maternal infection remained associated with a lower risk of brain injury, whereas the association between infant infection and NMS was no longer significant.

These preliminary results are in keeping with animal studies that suggest that the timing of an inflammatory signal may determine whether infection is injurious or protective.

Acknowledgements NIH UL1 RR024131\&P50NS035902.

\section{GENES IMPORTANT IN INFLAMMATION, APOPTOSIS, TRANSCRIPTION REGULATION AND ANGIOGENESIS ARE INDUCED IN THE NEWBORN MOUSE BRAIN AFTER HYPOXIA-REOXYGENATION (HR)}

doi:10.1136/archdischild-2012-302724.0290

1,2AGW Rognlien, 1,2EJ Wollen, ${ }^{2,3} \mathrm{M}$ Atneosen-Åsegg, ${ }^{4} \mathrm{M}$ Bjørås, 1,20D Saugstad. ${ }^{1}$ Department of Pediatric Research, Oslo University Hospital HF; ${ }^{2}$ Women and Children's Division, University of Os/o, Oslo; ${ }^{3}$ Department of Clinical Molecular Biology and Laboratory Sciences, Akershus University Hospital, Lørenskog; ' ${ }^{4}$ Department of Microbiology, Oslo University Hospital HF, Oslo, Norway

Background and Aims Pathogenesis of birth asphyxia has yet to be fully elucidated. To explore the mechanism of HR injury we followed the temporal profile of a priori selected genes in the newborn mouse brain

Methods 84 C57BL/6 mice (postnatal day 7) were randomized to 120 minutes of hypoxia $\left(\mathrm{FiO}_{2} 0.08, \mathrm{n}=64\right)$ or 180 minutes in air (controls (C21), $\mathrm{n}=20$ ). The hypoxia group was randomized to $30 \mathrm{~min}$ reoxygenation with $\mathrm{FiO}_{2} 0.60(\mathrm{H} 60)$ or air $\left(\mathrm{H}_{2} 1\right)$. After observation in air for $0,150,300$ minutes or 3 days, organs were harvested. Homogenate of hippocampus and striatum was analyzed for mRNA expression of 44 genes by real-time PCR.

Results Lcn2, Mt1, Hmox1 and Vegfa were significantly up-regulated $(\mathrm{p}<0.05)$ after $0-300$ min observation when comparing $\mathrm{H}_{2} 1 \mathrm{vsC21}$ and H60vsC21. Ccl2, Ccl12 and Tnf were up-regulated from 0-150 min, Stat3 from 150-300 min, while Ccnd1 was down-regulated at 0 min in both comparisons. In the $\mathrm{H}_{2} 1 \mathrm{vsC} 21$ comparison at 0 min, Neil3 and Apaf1 were down-regulated. When comparing H60vsH21, Cxcl10 (0 min) and Hmox1 (300 min) were up-regulated while Neil3 $(0 \mathrm{~min})$ was down-regulated. There were no significant gene expression changes after 3 days.

Conclusions Genes important in inflammation ( $L \mathrm{cn} 2, \mathrm{Mt1}, \mathrm{Ccl} 2$, Ccl12, Cxcl10, Tnf, Hmox1), apoptosis (Lcn2, Mt1, Tnf, Hmox1, Vegfa), angiogenesis (Vegfa), and transcription regulation (Stat3) were induced up to 300 minutes after hypoxia-reoxygenation while the DNA-glycosylase Neil3 was suppressed. The up-regulation of the pro-inflammatory cytokine Cxcl10 after hyperoxic compared to normoxic reoxygenation, confirms that hyperoxia induces additional inflammation.

\section{1 \\ PROGNOSTIC TESTS IN TERM NEONATES WITH HYPOXIC ISCHEMIC ENCEPHALOPATHY: A SYSTEMATIC REVIEW}

doi:10.1136/archdischild-2012-302724.0291

${ }^{1} \mathrm{H}$ van Laerhoven, ${ }^{2} \mathrm{TR}$ de Haan, ${ }^{3} \mathrm{M}$ Offringa, ${ }^{4} \mathrm{~B}$ Post, ${ }^{5} \mathrm{JH}$ van der Lee. ${ }^{1}$ Academic Medical Center - Emma Children's Hospital; ${ }^{2}$ Neonatology, Academic Medical Center Emma Children's Hospital, Amsterdam, Netherlands Antilles; ${ }^{3}$ Hospital for Sick Children Research Institute, Toronto, ON, Canada; ${ }^{4}$ UMCN St Radboud, Nijmegen; ${ }^{5}$ Woman and Child Centre, Academic Medical Centre, Amsterdam, Netherlands Antilles
Background and Aims Hypoxic Ischemic Encephalopathy (HIE) following perinatal asphyxia in term neonates is associated with long term neurological sequelae or demise. The aim of this systematic review was to investigate the prognostic value of currently used clinical tests in neonatal patients with HIE.

Methods MEDLINE, EMBASE, Central and CINAHL were searched until November 2011. Studies were included if they: (1) concerned infants with a gestational age $\geq 36$ weeks suffering perinatal asphyxia and HIE; (2) evaluated prognostic tests in either cooled or non-cooled patients; (3) reported on neurodevelopmental outcome results at a follow up age $\geq 18$ months. Study selection, assessment of methodological quality, and data extraction was performed by three independent reviewers. Pooled sensitivities and specificities of investigated tests were calculated when possible.

Results Included in the analysis were 29 studies describing 13 different prognostic tests conducted 1631 times in 1306 term neonates. Investigated tests comprised a range of imaging modalities, neurophysiological tests and clinical neurological exams. Most promising neurophysiology tests (first week of life) were: aEEC (sens. 0.93, [95\%CI 0.78-0.98]; spec. 0.90 [0.60-0.98]); EEG (sens. $0.92[0.66-0.99]$; spec. $0.83[0.64-0.93]$ ) and VEP (sens. 0.90 [0.74-0.97]; spec. 0.92 [0.68-0.98]).

Conclusions The available evidence suggests an important role for aEEG, EEG, and VEP. Given the heterogeneity of the tests' performance and outcomes studied, accurate predictions of long term outcomes in these critically ill neonates await the results of well designed large prospective studies that evaluate the best possible combination and timing of diagnostic tests.

\section{THE IMPACT OF HYPOTHERMIA ON POST-NATAL BLOOD BIOMARKERS OF NEONATAL HYPOXIC ISCHAEMIC ENCEPHALOPATHY}

doi:10.1136/archdischild-2012-302724.0292

BH Walsh, GB Boylan, EM Dempsey, DM Murray. Neonatal Brain Research Group, Department of Paediatrics and Child Health, University College Cork, Cork, Ireland

Numerous post-natal biomarkers of hypoxic ischaemic encephalopathy (HIE) severity have been proposed before the era of hypothermia. It is unclear if hypothermia impacts upon these potential biomarkers, and therefore whether previous findings are now valid. The aim of this study was to determine if hypothermia alters the discriminative ability of post-natal nucleated red blood cells (NRBCs) to distinguish between mild and moderate/severely encephalopathic infants.

Methods A prospective cohort study recruited term infants with HIE. The grade of HIE was categorised using Sarnat score, and multi-channel EEG. The recruitment period (2003-2012), spanned the introduction of hypothermia. Therefore the discriminative ability of the NRBC count for grade of encephalopathy could be compared in moderate/severely encephalopathic infants who did and did not receive hypothermia.

Results 86 infants with HIE were included in the study, 40 were mild, 26 moderate (14 normothermic, 12 hypothermic), and 18 severe (10 normothermic, 8 hypothermic). In the normothermic group, the NRBC count discriminated between mild and moderate/ severe Sarnat scores $(p=0.016)$, but not in the hypothermic group $(p=0.297)$. This change was due to a decrease in NRBCs among infants with a moderate Sarnat score receiving hypothermia, This occurred despite these infants having a significantly worse 5 minute Apgar score $(p<0.001)$ and background EEG at 6 hours $(p=0.032)$ than their normothermic counterparts.

Conclusion This study has demonstrated that hypothermia can impact upon early post-natal blood biomarkers of HIE. We therefore advise caution in the use of these samples when studying novel diagnostic biomarkers for HIE in the hypothermic era. 


\section{THE MYOCARDIAL PERFORMANCE DURING AND AFTER 72-HOURS OF THERAPEUTIC HYPOTHERMIA FOR PERINATAL ASPHYXIA IN TERM NEONATES}

doi:10.1136/archdischild-2012-302724.0293

${ }^{1} \mathrm{E}$ Nestaas, ${ }^{2,3} \mathrm{~J}$ Skranes, ${ }^{4,5} \mathrm{~A}$ Støylen, ${ }^{2,3} \mathrm{D}$ Fugelseth. 'Department of Paediatrics, Vestfold Hospital Trust, Tønsberg; 'Department of Neonatal Intensive Care, Os/o University Hospital, Ullevål; ${ }^{3}$ Faculty of Medicine, University of Oslo, Oslo; ${ }^{4}$ Department of Circulation and Medical Imaging, Faculty of Medicine, Norwegian University of Science and Technology; ${ }^{5}$ Department of Cardiology, St. Olavs Hospital, Trondheim, Norway

Background and Aims Therapeutic hypothermia after perinatal asphyxia reduces brain damage. The impact on the heart is poorly investigated. This study compare myocardial performance (MP) by ultrasound (Strain and Strain-Rate by tissue Doppler) in cooled asphyxiated term neonates (HT), asphyxiated term neonates treated with normothermia (NT) and non-asphyxiated term neonates (CTR).

Methods MP were compared in $44 \mathrm{HT}$ during and after cooling, 20 NT and 48 CTR.

Results The HT-group was more severely asphyxiated than the NTgroup (pH 7.07 (7.02, 7.12) (mean (95\%CI)) vs. 7.21 (7.14, 7.30), BaseExcess $-16.11 \mathrm{mmol} / \mathrm{L}(-17.8,-14.3)$ vs. $-9.3(-13.0,-5.6)(\mathrm{p}<0.05))$.
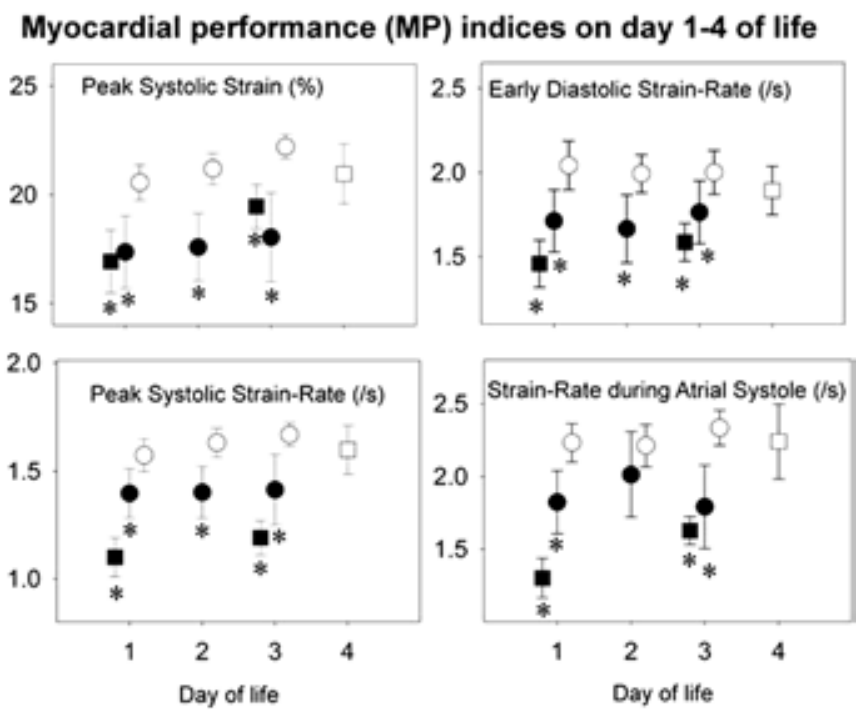

Absolute values (mean and $95 \%$ confidence intervals)

: Significantly different from the non-asphyxiated neonates $(p<0.05)$

Non-asphyxiated neonates (CTR group)

Asphyxiated normotherm neonates (NT group)

Asphyxiated neonates during therapeutic hypothermia (HT group)

Asphyxiated neonates after therapeutic hypothermia (HT group)

Abstract 293 Figure 1

On day 1-3, the MP was similar in the NT-group and HT-group during cooling, lower than in the CTR-group $(\mathrm{p}<0.05)$. The MP within each group was similar, except the CTR-group and HTgroup Peak Systolic Strain and HT-group Strain-Rate during the Atrial Systole $(\mathrm{p}<0.05)$.

After rewarming, the MP improved in the HT-group (day 4), approaching the MP in the CTR-group on day 3.

Conclusions Although the HT-group was more severely asphyxiated than the NT-group, the myocardial performance was similarly depressed on day 1-3. The myocardial performance in the HT-group improved after rewarming (day 4), approaching the level in the CTR-group on day 3 . Therapeutic hypothermia did not decrease the myocardial performance during treatment and might have had a positive impact after treatment.

\section{SUCCESSFUL IMPLEMENTATION OF HYPOTHERMIA TREATMENT IN SWEDEN - OUTCOME AT 24 MONTHS OF AGE IN HYPOTHERMIA-TREATED INFANTS WITH HIE}

doi:10.1136/archdischild-2012-302724.0294

K Robertson Grossmann, J Ragnö, B Vollmer, M Blennow, B Hallberg. Neonatology, Karolinska Institute, Karolinska University Hospital, Stockholm, Sweden

Background Hypoxic-ischemic encephalopathy (HIE) is associated with a high risk for subsequent neurological sequele. This study evaluates the implementation of HT in Sweden. Are national guidelines adhered to? Are outcome results comparable to those from previous controlled trials?

Methods Between 2007-2009, HT was available at 8 Swedish hospitals. Outcome data regarding all 187 infants who received HT during this period was collected and scored (normal/"motor delay"/ cerebral palsy (CP)/dead). Local guidelines for clinical follow-up differing from the national guidelines were also reviewed.

Results

Abstract 294 Table 1

\begin{tabular}{ll}
\hline Outcome & $\mathbf{N = 1 6 3 / 1 8 7}$ (24 children lost but alive) \\
\hline Normal & $109(58 \%)$ \\
"Motor delay" & $10(5 \%)$ \\
Cerebral palsy & $20(11 \%)$ \\
Dead & $24(13 \%)$ \\
\hline
\end{tabular}

Outcome data was available in $87 \%$ of treated infants. $13 \%$ had died, 74\% had been assessed between 15-36 months of age with a neurological exam and using scales for grading of severity of $\mathrm{CP}$, such as the Gross Motor Classification System. 35\% had been assessed according to national guidelines using Bayley III

Conclusions HT has been successfully implemented; however structured, comprehensive assessments according to national guidelines are not always carried out. In particular, only about $1 / 3$ of infants undergo a psychometric developmental assessment. The incidence of severe adverse neuromotor outcome is comparable to what was to be expected from earlier RCTs, showing that HT has a significant positive effect also outside of controlled trials.

\section{ROLE OF ANAPYREXIA IN PERINATAL ASPHYXIA IN TERM NEWBORNS: AN OPEN LABELED RANDOMIZED CONTROLLED TRIAL}

doi:10.1136/archdischild-2012-302724.0295

A Garg, A Saili, S Nangia. Department of Pediatrics, Kalawati Saran Children's Hospital, Delhi, India

Background and Aims To compare anapyrexia (a physiological decrease in body temperature in neonates in response to hypoxia) with normothermia (conventional management) with respect to immediate outcome and neuro-developmental outcome at 3 and 6 months of age in full term asphyxiated newborns.

Methods Fifty full term intramural neonates with severe birth asphyxia were randomized to Study (Anapyrexia) group and Control (Normothermia) group. Neonates in Anapyrexia group were allowed to remain at their own intrinsic temperature for a period of 72 hours. Neonates randomized to the Normothermia group had their temperature maintained between $36.5^{\circ} \mathrm{C}$ to $37.5^{\circ} \mathrm{C}$ with assistance from the servocontrolled radiant warmer. All neonates were managed according to standard management protocol of the unit. The subjects were monitored closely for any adverse effects, seizures, and development of HIE. The neurological examination was done at 6 hourly interval for the first 24 hours and then at 12 hourly interval. The surviving neonates were followed up at 3 months and 6 months of age. 